\title{
Correction to: Learning from the past: did experience with previous epidemics help mitigate the impact of COVID-19 among spine surgeons worldwide?
}

\author{
Joseph A. Weiner ${ }^{1}$ • Peter R. Swiatek ${ }^{1}$ - Daniel J. Johnson ${ }^{1}$ - Philip K. Louie ${ }^{2} \cdot$ Garrett K. Harada $^{3,4}$. \\ Michael H. McCarthy ${ }^{2}$. Niccole Germscheid ${ }^{5}$. Jason P. Y. Cheung ${ }^{6}$. Marko H. Neva ${ }^{7}$. Mohammad El-Sharkawi ${ }^{8}$. \\ Marcelo Valacco ${ }^{9}$. Daniel M. Sciubba ${ }^{10}$. Norman B. Chutkan ${ }^{11} \cdot$ Howard S. An ${ }^{3,4}$. Dino Samartzis ${ }^{3,4}(1)$
}

Published online: 7 September 2020

(c) Springer-Verlag GmbH Germany, part of Springer Nature 2020

\section{Correction to: European Spine Journal (2020) 29:1789-1805 https://doi.org/10.1007/s00586-020-06477-6}

Unfortunately, the 13th author name has been incorrectly published in the original publication. The complete correct name is given below.

Norman B. Chutkan
Publisher's Note Springer Nature remains neutral with regard to jurisdictional claims in published maps and institutional affiliations.

The original article can be found online at https://doi.org/10.1007/ s00586-020-06477-6.

Dino Samartzis

Dino_Samartzis@rush.edu

1 Department of Orthopaedic Surgery, Northwestern University, Chicago, IL, USA

2 Department of Orthopaedic Surgery, Hospital for Special Surgery, New York, NY, USA

3 Department of Orthopaedic Surgery, Rush University Medical Center, Orthopaedic Building, Suite 204-G, 1611 W Harrison Street, Chicago, IL 60612, USA

4 The International Spine Research and Innovation Initiative, Rush University Medical Center, Chicago, IL, USA

5 Research Department, AO Spine International, Davos, Switzerland
6 Department of Orthopaedics and Traumatology, The University of Hong Kong, Hong Kong, China

7 Department of Orthopaedic and Trauma Surgery, Tampere University Hospital, Tampere, Finland

8 Department of Orthopaedic and Trauma Surgery, Assiut University Medical School, Assiut, Egypt

9 Department of Orthopaedics, Churruca Hospital de Buenos Aires, Buenos Aires, Argentina

10 Department of Neurosurgery, John Hopkins University, Baltimore, MD, USA

11 Department of Orthopaedic Surgery, University of Arizona College of Medicine, Phoenix, AZ, USA 